
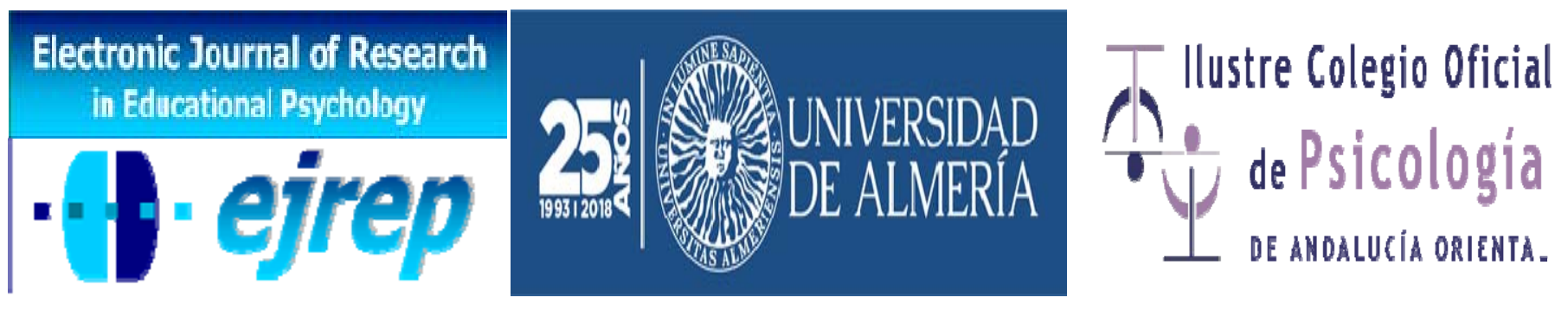

\title{
Relación entre las funciones ejecutivas y la empatía y su influencia en el rendimiento académico en alumnado de Formación Profesional Básica
}

\author{
Sandra Cid-Sillero ${ }^{1}$, Sandra Santiago-Ramajo. ${ }^{2}$ y María Pilar \\ Martín-Lobo ${ }^{2}$ \\ ${ }^{1}$ Instituto Municipal de Formación Profesional Básica de Ermua-Mallabia. Área \\ de desarrollo local. Ayuntamiento de Ermua (Bizkaia). \\ ${ }^{2}$ Universidad Internacional de la Rioja (UNIR). Facultad de Educación. Máster \\ de Neuropsicología y Educación (La Rioja).
}

\section{España}

Correspondencia: Sandra Santiago-Ramajo (PhD). Universidad Internacional de la Rioja (UNIR). Av. de la Paz, 137. Logroño 26006, La Rioja, España. E-mail: sandra.sramajo@unir.net 


\section{Resumen}

Introducción. Una de las líneas de investigación que mayor interés ha despertado durante estos últimos años ha sido determinar el papel que juegan ciertas habilidades cognitivas en el desempeño académico. Este artículo describe la relación existente entre las funciones ejecutivas (interferencia, flexibilidad y planificación), la empatía y su influencia en el rendimiento académico de un grupo de alumnos que estudia en un instituto de Formación Profesional Básica (FPB).

Método. La muestra estuvo compuesta por un total de 80 alumnos/as entre los 15 y los 19 años de edad. Los instrumentos empleados fueron: STROOP (interferencia), Trail Making Test (TMT) (flexibilidad), Torre de Hanoi (planificación) y TECA (Test de Empatía Cognitiva y Afectiva). La variable rendimiento académico fue medida a partir de los resultados obtenidos en el ámbito sociolingüístico y en el científico-tecnológico (calificaciones de las distintas asignaturas). Se aplicaron correlaciones de Pearson y regresiones múltiples.

Resultados. Los resultados indican que no existe una relación significativa entre las funciones ejecutivas y la empatía. El 15\% de la variabilidad del rendimiento académico se puede explicar mediante el nivel de las funciones ejecutivas. En cambio, la empatía no influye en el rendimiento académico.

Discusión y conclusiones. El estudio demuestra la importancia de contemplar el desarrollo de las funciones ejecutivas en los/as adolescentes que cursan la FPB y determinar su influencia en el rendimiento académico.

Palabras Clave: funciones ejecutivas, empatía, formación profesional básica, rendimiento académico. 


\begin{abstract}
Introduction. One of the lines of research that has aroused great interest in recent years has been to determine the role that certain cognitive abilities play in academic performance. This article describes the relationship between executive functions (interference, flexibility and planning), empathy and its influence on the academic performance of a group of students studying a Basic Vocational Education and Training (BVET) College.
\end{abstract}

Method. A total of 80 students between the age of 15 and 19 participated in the study. The instruments used were: STROOP (interference), Trail Making Test (TMT) (flexibility), Tower of Hanoi (planning) and CAET (Cognitive and Affective Empathy Test). The Academic Performance variable was measured from results obtained from the Sociolinguistic and Scientific-Technological fields. Pearson correlations and multiple regressions have been applied.

Results. The results show that no significant correlations were found between executive functions and affective empathy, $15 \%$ of the variance of academic achievement can be explained by executive functions and that affective empathy has no significant influence on academic performance.

Discussion and Conclusion: This study underlines the importance of taking into account the level of executive functions in adolescent BVET students in relation to academic performance.

Keywords: executive functions, empathy, basic vocational education, academic performance. 


\section{Introducción}

La neurociencia y la educación a lo largo de estas últimas décadas parecen aunar esfuerzos para lograr una praxis orientada al desarrollo integral del alumnado. No obstante, hallar puntos de convergencia entre las teorías que describen los procesos cognitivos implicados en el aprendizaje y la práctica educativa en el marco del sistema educativo actual, puede resultar una ardua tarea. Se trata de un gran reto que la comunidad educativa debe asumir como propio si quiere dar respuesta al nuevo perfil del alumnado.

Actualmente en España, están surgiendo una serie de retos sociales que están creando tendencia en el nuevo perfil del alumnado. Olmos (2014) advierte acerca de su importancia y describe las causas de su bajo rendimiento académico en el contexto escolar. Según éste, existen factores personales, sociofamiliares, psicológicos, de apoyo social, de adaptación, de estilo de aprendizaje y de estilo docente, que dan respuesta a la conducta del alumnado en el ámbito educativo. El alumnado de secundaria que presenta dificultades para adaptarse a las exigencias de la educación ordinaria o que presenta problemas de disciplina que han derivado en una falta importante de motivación, de interés y de autoestima, es porque parte de una mala experiencia educativa previa (Ritacco y Amores, 2015). Y es que, según Diamond (2013), uno de los componentes más importantes para que el desarrollo infantil y adolescente sea exitoso son las funciones ejecutivas. Dichas funciones y la atención son el eje motor de la actividad intelectual y de las respuestas emocionales, ya que, gracias a éstas, nuestras acciones son el resultado de nuestros pensamientos (Portellano, 2014).

\section{Empatía y afectividad}

La empatía y la afectividad como componentes constitutivos de la inteligencia emocional, bajo el modelo de Salovey y Mayer (Ibarrola, 2013), parecen estar relacionados con el ajuste socio-escolar del alumnado (Jiménez y López, 2009). La inteligencia emocional es como un cajón de sastre que aporta una serie de habilidades psicológicas influyentes en la conducta del alumnado. Estas habilidades tienen que ver con el autocontrol, el estado de ánimo, el entusiasmo, la automotivación, la empatía, la autoconciencia, la perseverancia, la agilidad mental o la capacidad para interaccionar con el resto (García, Tirapu, Luna, Ibañez y Duque, 2010). Dicho tipo de inteligencia resulta imprescindible debido a su proyección en la vida cotidiana, ya que consiste en encontrar el equilibrio permanente entre la mente racional (lóbulo frontal) y la mente emocional (sistema límbico). También el control cognitivo es muy 
importante en la toma de decisiones socio-afectivas (Prencipe, Kesek, Cohen, Lamm, Lewis y Zelazo, 2011).

La literatura sugiere que aquellos adolescentes con una mayor empatía y capacidad de regulación emocional se adaptan mejor a su entorno social (Salguero, Fernández, Ruiz, Castillo y Palomera, 2011). También evidencia la relación existente entre la dimensión cognitiva y la dimensión afectiva de la empatía con la inteligencia emocional, el género y la edad, resultando presentar un mayor nivel de empatía el género femenino. No obstante, Gorostiaga, Balluerka y Soroa (2013) en su investigación no encontraron correlaciones significativas en relación a la edad.

El Sistema Educativo Español siendo consciente de su dificultad para llegar a todo el alumnado y a través de la Ley Orgánica 8/2013, de 9 de diciembre, para la mejora de la calidad educativa (LOMCE, 2013) en el preámbulo IV, apoya un modelo pedagógico que integre el desarrollo cognitivo del alumnado y contribuya a erradicar la exclusión educativa y el fracaso escolar logrando una mayor capacitación del alumnado mediante la adquisición de competencias transversales para superar satisfactoriamente dicha etapa a través de la Formación Profesional Básica (FPB).

Se trata de una formación Profesional de Nivel I según el Catálogo Nacional de Cualificaciones Profesionales y se complementa con una formación en cultura general. $\mathrm{Su}$ finalidad es favorecer la inserción social, educativa y laboral de jóvenes que comprenden principalmente los 15 y 17 años de edad y que abandonan el sistema educativo sin el título de Educación Secundaria Obligatoria. Se organiza en dos cursos y en torno a tres módulos: los módulos específicos, el módulo de orientación y tutoría y los módulos de carácter general. Las asignaturas que se imparten en estos últimos módulos están agrupadas en dos ámbitos: el ámbito sociolingüístico (Lengua castellana, Euskera, Inglés y Ciencias Sociales) y el ámbito científico-tecnológico (Matemáticas y Ciencias Naturales).

Dichos programas, contribuyen a garantizar que la formación, las oportunidades y los resultados estén al margen de la situación socioeconómica del alumnado, así como de otros factores que puedan generar en éste cierta desventaja educativa, tales como la discapacidad, la emigración, la exclusión social, la desigualdad de oportunidades, etc. Sin embargo, para los adolescentes, el éxito de lograr una meta académica implica motivación y el deseo de superar 
las dificultades. La adolescencia es una etapa de transición entre la infancia y la adultez, se trata de un momento natural de aprendizaje y de ajuste, por ello, las motivaciones están influenciadas por sus experiencias sociales y relacionadas con sus propios sentimientos y prioridades con respecto a esa meta. Además, la adolescencia es una etapa de especial vulnerabilidad por el desarrollo gradual de las funciones ejecutivas (Crone y Dahl, 2012).

\section{Funciones ejecutivas}

Bausela (2014) apoyado en el modelo propuesto por Miyake, Friedman, Emerson, Witzki, Howerter y Wager (2000) defiende la independencia de tres componentes de las Funciones ejecutivas: la memoria de trabajo, la flexibilidad y la respuesta inhibitoria, siendo fácilmente operacionalizadas y pudiendo ser estudiadas usando tareas comunes. La planificación es otra de las funciones ejecutivas más importantes por su componente práctico para el desenvolvimiento en la vida cotidiana. Se cree que las funciones ejecutivas frías comprenden la memoria de trabajo, el control inhibitorio y la flexibilidad cognitiva, las cuales permiten activar un pensamiento y comportamiento consciente dirigido siempre a una meta (Prencipe et al., 2011). Las funciones calientes, sin embargo, implican estímulos, decisiones y motivaciones asociadas a lo emocional y lo afectivo. Otras líneas de investigación reconocen la interacción entre ambas a la hora de actuar y tomar decisiones (Cunningham y Zelazo, 2007); no obstante, las funciones calientes parecen desarrollarse de forma más tardía que las frías en la etapa de la adolescencia y los estímulos emocionalmente significativos parecen interferir en el control cognitivo durante la adolescencia (Crone y Dahl, 2012).

Esta investigación estudia las variables interferencia, flexibilidad cognitiva y planificación, dado que nos permiten organizar y planificar nuestra conducta, así como programar las secuencias necesarias para lograr un objetivo. Gracias a dichas funciones, el ser humano es capaz de inhibir la distracción y evitar la interferencia de los estímulos irrelevantes, regulando los procesos atencionales (Portellano, 2014). Además, la interferencia en el desarrollo del control inhibitorio permite al alumnado realizar tareas mentales que requieren procesar información que compite entre sí empleando el procedimiento óptimo para resolver una tarea (Flores, Castillo y Jiménez, 2014). La flexibilidad cognitiva, permite al alumnado generar una respuesta alternativa, enfrentándose de forma eficiente a la solución de un problema y la planificación, posibilita avanzar en la organización de sus actividades escolares y acciones personales, pudiendo establecer prioridades y una sucesión de acciones encaminadas a una meta (Flores et al., 2014). Según Pérez y Beltrán (2014), algunos de los 
síntomas que respaldan el bajo rendimiento académico son entre otros, la poca flexibilidad cognitiva, la baja memoria de trabajo del alumnado, la baja inhibición conductual, las estrategias de aprendizaje, la baja motivación y la dificultad en la regulación emocional del alumnado. A nivel emocional, se evalúa la variable empatía en el contexto educativo a través de la comprensión de los puntos de vista ajenos (Adopción de perspectivas), el estrés empático y la capacidad de sintonizar con las alegrías y éxitos ajenos (Alegría empática).

La empatía tiene que ver con la capacidad de comprender los sentimientos y emociones de los demás y se trata de una habilidad indispensable para los adolescentes dado que su vida transcurre en contextos sociales complejos (López, Arán y Richaud, 2014). No obstante, aunque es posible individualizar cada componente asociado al concepto de empatía, en el funcionamiento normal, están todos interrelacionados e implicados en la cognición social. Algunas líneas de investigación sobre el tema han encontrado que existe una relación entre el estrés escolar y la empatía; es decir, cuando hay menor estrés escolar, se da mayor empatía o el grupo favorece una convivencia con más capacidad de comprensión de emociones entre estudiantes (Sierra, Urrego, Montenegro y Castillo, 2015).

\section{Objetivos e hipótesis}

La presente investigación tiene como objetivo determinar la relación existente entre las funciones ejecutivas, la empatía y su influencia en el rendimiento académico del alumnado de FPB. De manera concreta, los objetivos específicos que persigue la investigación son: 1) Estudiar la relación entre las funciones ejecutivas (interferencia, flexibilidad y planificación) y la empatía (adopción de perspectivas, comprensión emocional, estrés empático, alegría empática). 2) Estudiar la influencia de las funciones ejecutivas en el rendimiento académico (ámbito sociolingüístico y científico-tecnológico). 3) Estudiar la influencia de la empatía en el rendimiento académico. La hipótesis de partida del estudio es que las funciones ejecutivas y la empatía están relacionadas y que las funciones ejecutivas y la empatía influyen significativamente en el rendimiento académico.

\section{Método}

\section{Participantes}

La muestra estuvo compuesta por un total de 80 estudiantes pertenecientes al primer y segundo curso del Instituto de Formación Profesional Básica de Ermua-Mallabia, centro que 
anualmente abre sus puertas a un número aproximado de 90 alumnos/as procedentes de dos, de las tres provincias que constituyen la Comunidad Autónoma del País Vasco (Bizkaia y Gipuzkoa) y que representan cerca de 20 municipios diferentes. Del total la muestra, cerca de un $96 \%$ vivía con algún familiar y un $4 \%$ procedía de centros o familias de acogida. El 31.3\% del alumnado fueron chicas $(n=25)$ y el $68.8 \%$ chicos $(n=55)$, representando este último la mayoría de la muestra debido a que el centro oferta tres especialidades diferentes: Auxiliar de peluquería y estética (24 estudiantes, 14 en el primer curso y 12 en segundo) Auxiliar de mantenimiento de vehículos (28 estudiantes, 16 en el primer curso y 12 en segundo) y Operario en fabricación de elementos metálicos (28 estudiantes, 16 en primero y 12 en segundo). Ninguno de los participantes era repetidor. A pesar de que en peluquería estuviesen matriculados dos chicos y en reparación de vehículos dos chicas, suele ser común que la mayoría del alumnado que cursa la especialidad de reparación de vehículos y la de fabricación de elementos metálicos, sean chicos. La edad del alumnado osciló entre los 15 y los 19 años $(\mathrm{M}=16.58$, DT=1.07). Cabe destacar que cada asignatura fue evaluada por su profesor correspondiente, pero el sistema de evaluación fue el mismo en todas las asignaturas.

\section{Instrumentos}

Las variables medidas en el presente estudio han sido tres principalmente: funciones ejecutivas (interferencia, flexibilidad y planificación), empatía (adopción de perspectivas, comprensión emocional, estrés empático, alegría empática) y rendimiento académico (ámbito sociolingüístico y científico-tecnológico). Para su medición, se han empleado cuatro pruebas: Stroop (para medir interferencia), Trail Making Test (TMT) (para medir flexibilidad), Torre de Hanoi (para medir planificación) y Test de Empatía Cognitiva y Afectiva (TECA) (para medir empatía):

Test de colores y palabras STROOP (Delis, Kaplan y Kramer, 2001; Charles \& Golden, 1994). El test de Stroop es una prueba atencional que evalúa la habilidad para resistir la interferencia verbal. Se trata de un test de aplicación individual y su duración es de 5 minutos. Consta de 3 láminas impresas que contienen cinco columnas de 20 elementos separadas entre sí por tres centímetros. Inicialmente, el alumno lee en voz alta la primera lámina denominada "lectura de palabras (P)" durante 45 segundos, que contiene el nombre de los tres colores (rojo, verde y azul) utilizados en el test repetido de manera aleatoria e impresos en tinta negra. Posteriormente, lee la lámina dos, denominada "lectura de colores (C)" también durante 45 segundos; está constituida por cinco columnas de símbolos tipo "XXX" coloreados de forma 
aleatoria con los mismos tres colores antes mencionados. Por último, el alumno lee la tercera y última lámina denominada "Palabras-Colores (PC)" durante 45 segundos, en la que aparece el nombre de los tres colores empleados en el test pero impresos en tinta de manera aleatoria y sin concordancia entre el nombre del color y el color de la tinta en que está impreso. Tras la medición de estos tres índices P, C y PC, que indican el número de palabras que el alumno lee correctamente durante los mencionados 45 segundos, se calcula la variable interferencia de la siguiente manera: $\mathrm{PC}=($ palabras $(\mathrm{P}) \mathrm{x}$ colores $(\mathrm{C})) /($ palabras $(\mathrm{P})+$ colores $(\mathrm{C}))$. Por último, la resta entre la puntuación que realmente obtiene (PC) y la estimación de la que debería obtener (PC"), es el indicador que informa de cuánto se deja interferir el alumno por el efecto Stroop. La fiabilidad del test-retest STROOP es bastante consistente, ya que oscila entre el .71 y el .89 para las puntuaciones directas de cada una de las tres láminas.

The Trail Making Test (TMT) (Army Individual Test Battery, 1944). El Trail Making Test es una prueba que mide la flexibilidad cognitiva (flexibilidad mental y velocidad de procesamiento). Consta de dos partes (A y B). La parte A consiste en unir 25 números con el lápiz realizando un trazo del uno al otro en orden, lo más rápido posible y sin levantar el lápiz del papel. La parte $\mathrm{B}$ consiste en unir los doce números y las doce letras expuestas aleatoriamente en la hoja de la forma más rápida posible y sin levantar el lápiz del papel, alternando una letra y un número y respetando el orden de sucesión de los números y el orden alfabético de las letras. El evaluador mide el tiempo (en segundos) que tarda la persona en realizar cada una de las partes de la prueba (A y B). La variable medida se extrae de la siguiente fórmula: segundos de la parte $\mathrm{B} /$ segundos de la parte $\mathrm{A}$. La consistencia interna del instrumento es de .70 . Su fiabilidad interjueces oscila entre el .96 y el .98 , resultando la fiabilidad del test-retest entre el $.70 \mathrm{y}$ el .78 para las puntuaciones directas.

Prueba de la Torre de Hanoi (León, 1997). Esta prueba mide la planificación cognitiva y aunque existen varias versiones de la misma, ha sido administrada en formato digital. Consta de 3 torres o varillas verticales y tres discos de diferentes tamaños apilados de mayor a menor radio en la primera torre, quedando las otras dos vacantes. La prueba consiste en pasar todos los discos de la torre ocupada a la tercera y última, respetando en ésta el mismo orden que tenían los discos inicialmente. Para ello, hay que mover los discos de uno en uno: un disco mayor no puede descansar sobre un disco más pequeño y solamente se puede mover el disco que se encuentra arriba en cada torre. La variable medida es el número de 
movimientos que necesita la persona para pasar los discos de la torre uno, a la torre tres; su fiabilidad según el alpha de Cronbach es de .72.

Test de Empatía Cognitiva y Afectiva (TECA) (López-Pérez, Fernández-Pinto y Abad, 2008). Es un cuestionario que mide la empatía y la afectividad mediante 4 escalas: Adopción de perspectivas (A.P), Comprensión emocional (C.E), Estrés empático (E.E) y Alegría empática (A.E). Se trata de un test compuesto por 33 ítems (pertenecientes a las escalas mencionadas). A través de una escala tipo Likert, el alumnado debe asignar una puntuación del 1 al 5 según su grado de conformidad con el ítem (1=Totalmente en desacuerdo5=Totalmente de acuerdo). Posteriormente, se debe anotar en la plantilla de respuestas la puntuación asignada a cada ítem. Para calcular las puntuaciones de las cuatro escalas del test, se debe copiar la puntuación de cada ítem en el recuadro sombreado que aparece en la misma fila a la izquierda. Posteriormente, se suman las puntuaciones de los recuadros por columnas y se anota el resultado en la casilla correspondiente a la puntuación directa (PD) de cada escala: A.P (18-31), C.E (22-38), E.E (17-28) y A.E (19-37). Para calcular la puntuación total en el test, hay que sumar la puntuación de las cuatro escalas y anotar el resultado en la casilla de puntuación directa total (PD total 88-124). El alfa de Cronbach es de .86 para el TECA global y oscila entre el .70 y el .78 para las cuatro dimensiones (A.P, C.E, E.E y A.E).

Rendimiento académico. Se han registrado las calificaciones finales del curso en los dos ámbitos de conocimiento. El ámbito sociolingüístico está compuesto por las asignaturas de Lengua Castellana, Euskera, Inglés y Ciencias Sociales. El ámbito científico-tecnológico está compuesto por las asignaturas de Matemáticas y Ciencias Naturales. La puntuación obtenida es la calificación final que ha obtenido el alumno en cada ámbito y está recogida en una escala de 0 a 10.

\section{Procedimiento}

Antes de comenzar con la aplicación de las pruebas, se solicitó el permiso correspondiente a la dirección del centro dado que la aplicación de las pruebas, supondría una implicación importante del alumnado, sobre todo, en inversión de tiempo. También se informó al equipo docente, al cual se le solicitó ayuda para organizar a las personas participantes con el objetivo de interferir lo menos posible en la vida del centro y en el ritmo de las clases. El alumnado que constituye la muestra fue autorizado a participar en el presente 
estudio mediante la firma de un consentimiento informado por parte de sus responsables legales.

Las variables (interferencia, flexibilidad y planificación) fueron medidas de manera individual a lo largo de cuatro semanas durante el mes de mayo. El tiempo medio que cada alumno requirió para completar las tres pruebas fue de unos 35 minutos aproximadamente. Para la aplicación de los instrumentos, se habilitó un espacio libre de ruidos (favoreciendo así la concentración del alumnado) y con todas las herramientas necesarias (ordenador, pruebas impresas, bolígrafos, cronómetro) para su ejecución. Las instrucciones fueron uniformes para todo el alumnado resultando breves, claros y concisos. Para la medición de la variable empatía, se empleó el Test de Empatía Cognitiva y Afectiva. Dicho test, fue aplicado en la hora de orientación y tutoría. La prueba se llevó a cabo bajo un riguroso procedimiento, creando un clima de seguridad, tranquilidad y confianza con el alumnado, favoreciendo un ambiente silencioso, reflexivo, asegurando su comprensión y evitando la presión del tiempo. La duración máxima de la prueba fue de 60 minutos. Para la medición de la última variable (rendimiento académico) fue necesario esperar a la finalización del curso (junio), momento en el que se celebraron las reuniones de evaluación. A partir de los boletines, se recogieron las calificaciones en cada una de las materias que componen los dos ámbitos académicos estudiados (sociolingüístico y científico-tecnológico).

\section{Análisis de datos}

Para la descripción de la muestra, se empleó la media y la desviación típica en la edad, así como la frecuencia (N) y el porcentaje (\%) en el sexo de las personas participantes. El análisis estadístico fue realizado mediante el programa Statistical Package for Social Sciences (SPSS, versión 21). En el objetivo 1, se aplicaron correlaciones de Pearson (ya que cumplían todos los supuestos para el análisis paramétrico). Debido al alto número de correlaciones aplicadas en el objetivo 1 (15 correlaciones) se consideró necesario aplicar un ajuste de Bonferroni $(.05 / 15=.003)$, por lo que se consideró significativo a $p<.003$. Para los objetivos 2 y 3 fueron aplicadas regresiones múltiples (método de 2 pasos). En las regresiones fueron incluidas las siguientes variables: el rendimiento académico como variable dependiente (realizando una regresión diferente para cada ámbito educativo: sociolingüístico y científicotecnológico) y las tres variables medidas en las funciones ejecutivas (interferencia, flexibilidad y planificación) como variable independiente del objetivo 2 y las cuatro subescalas de empatía como variable independiente del objetivo 3. Debido a que la muestra 
presentaba un rango de edad amplio (15 a 19 años), se decidió incluirla en el análisis de regresión para ver qué influencia podía tener esta variable en el rendimiento académico, aunque no formó parte como variable de estudio. La variable edad fue incluida en el primer paso de todas las regresiones y se calculó el cambio en $\mathrm{R}^{2}$ (variables de control). En el paso 2, se incluyeron todas las variables de interés para el estudio. Se realizaron 2 regresiones para el objetivo 2 (una para cada campo académico) y 2 regresiones para el objetivo 3 (una para cada campo académico). Para las regresiones se considera el nivel de significativa a .05 .

\section{Resultados}

\section{Correlación entre funciones ejecutivas y empatía}

Como se observa en la Tabla 1, no existe correlación estadísticamente significativa entre las tres variables que miden las funciones ejecutivas y el total de escala para empatía cognitiva y afectiva $(p>.003)$. Tampoco se encontraron correlaciones significativas entre las diferentes subescalas de empatía afectiva y las 3 medidas de las funciones ejecutivas.

Tabla 1. Correlaciones ( $r$ ) entre funciones ejecutivas y empatía afectiva, Media y Desviaciones Estándar

\begin{tabular}{lccccc}
\hline Variable & InterferenciaFlexibilidadPlanificación & $M$ & $D E$ \\
\hline Adopción de perspectivas & .00 & .11 & -.11 & 25.63 & 4.15 \\
Comprensión emocional & -.33 & .03 & -.01 & 30.75 & 3.84 \\
Estrés empático & -.04 & .08 & -.13 & 22.32 & 4.31 \\
Alegría empática & -.15 & .10 & .06 & 28.63 & 3.50 \\
TOTAL empatía afectiva & -.18 & .05 & -.08 & 107.35 & 10.86 \\
M & -.14 & 3.33 & 13.57 & & \\
DE & 7.12 & 1.36 & 6.46 & & \\
\hline
\end{tabular}

Nota: $* p<.003$

\section{Influencia de las funciones ejecutivas en el rendimiento académico}

La Tabla 2 muestra los resultados obtenidos en las regresiones múltiples. Se puede observar que las 3 variables de las funciones ejecutivas (interferencia, flexibilidad y 
planificación) pudieron predecir un 15\% de la variabilidad del rendimiento académico (en el ámbito sociolingüístico y científico-tecnológico) $(p<.05)$, eliminando el efecto de la edad. La edad pudo representar entre el $6 \%$ y el $9 \%$ de la variable de rendimiento académico (los sujetos mayores obtuvieron puntuaciones más altas en el rendimiento académico, $\beta=.30 \mathrm{y} \beta$ $=.25)$.

En referencia a la variable interferencia, los valores de $\beta$ indican que tuvo una influencia positiva en el desempeño académico en ambos campos (sociolingüístico y científico-tecnológico), lo que indica que los estudiantes con mayor capacidad para resistir interferencias, fueron precisamente aquellos con mejores calificaciones en sendos ámbitos $(\beta$ $=.35 \mathrm{y} \beta=.26$, respectivamente). La variable flexibilidad sólo tuvo una capacidad predictiva significativa en el campo científico-tecnológico $(\beta=-.18)$, lo que indica que los estudiantes con puntuaciones más altas en la prueba de flexibilidad (y por lo tanto menor capacidad de flexibilidad) presentaron puntuaciones más bajas. Por último, en la variable de planificación, se encontró una influencia significativa en el campo científico-tecnológico $(\beta=-.21)$, los estudiantes que necesitaron un mayor número de movimientos para completar la prueba obtuvieron una puntuación más baja en este ámbito.

En resumen, se encontró una capacidad predictiva significativa entre los puntajes en el campo científico-tecnológico y las tres variables de las funciones ejecutivas, mostrando que los estudiantes con puntajes más altos fueron los menos afectados por la interferencia, fueron más flexibles cognitivamente y tuvieron mejor capacidad de planificación. Sin embargo, en el campo Sociolingüístico, la única correlación significativa observada fue la variable de interferencia (Tabla 2).

Tabla 2. Análisis de regresión múltiple sobre la influencia de las funciones ejecutivas en el rendimiento académico.

\begin{tabular}{lcccccc}
\hline & \multicolumn{5}{c}{ Rendimiento académico } \\
\hline & Ámbito SociolingüísticoÁmbito Científico-Tecnológico \\
\hline Predictor & $\Delta \mathrm{R}^{2}$ & $\beta$ & $\mathrm{R}^{2}$ Change & $\Delta \mathrm{R}^{2}$ & $\beta$ & $\mathrm{R}^{2}$ Change \\
\hline $\begin{array}{l}\text { Step 1 } \\
\text { Control de variables (edad) }\end{array}$ & $.08^{* *}$ & & $.30^{* *}$ & $.09^{* *}$ & $.05^{* *}$ & \\
\hline
\end{tabular}




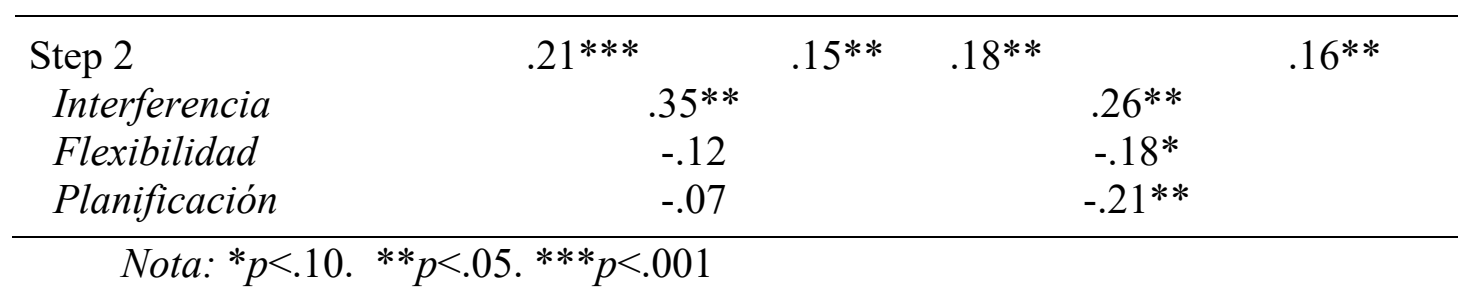

\section{Influencia de la empatía en el rendimiento académico}

Con la aplicación de regresiones múltiples (Tabla 3), las mediciones de empatía afectiva no fueron claramente predictivas del desempeño académico en ninguno de los dos campos $(p>.05)$. Al igual que en el objetivo anterior, se encontró una influencia significativa en la variable edad, lo que permitió predecir una variable adicional de 6-9\% en el rendimiento académico $(\beta=.30$ y $\beta=.25)$.

Tabla 3. Análisis de regresión múltiple sobre la influencia de la empatía afectiva en el rendimiento académico.

Rendimiento académico

\begin{tabular}{lcccccc}
\hline \multicolumn{5}{c}{ Ámbito SociolingüísticoÁmbito Científico-Tecnológico } \\
\hline Predictor & $\Delta \mathrm{R}^{2}$ & $\beta$ & $\mathrm{R}^{2}$ Change & $\Delta \mathrm{R}^{2}$ & $\beta$ & $\mathrm{R}^{2}$ Change \\
\hline Step 1 & $.08^{* *}$ & & $.09^{* *}$ & $.05^{* *}$ & & $.06^{*}$ \\
Control de variables (edad) & $.30^{* *}$ & & & $.25^{* *}$ & \\
\hline Step 2 & $.07^{*}$ & & .03 & .03 & & .03 \\
Adopción de perspectivas & & .02 & & & .10 & \\
Comprensión emocional & -.10 & & & -.20 & \\
Entré empático & .14 & & & .06 & \\
Alegría empática & .09 & & & -.02 & \\
\hline$\quad$ Nota: ${ }^{*} p<.10 .{ }^{* *} p<.05 .{ }^{* * *} p<.001$ & & &
\end{tabular}

\section{Discusión y Conclusiones}

Aunque no se ha encontrado correlación estadísticamente significativa entre las variables que miden las funciones ejecutivas (interferencia, flexibilidad y planificación) y el total de la escala de empatía cognitiva y afectiva (adopción de perspectivas, comprensión emocional, estrés empático, alegría empática), se ha encontrado que las funciones ejecutivas pueden predecir un $15 \%$ de la variabilidad del rendimiento académico, indicando la influencia 
que tienen los 3 componentes de las funciones ejecutivas estudiadas sobre el rendimiento académico. Los alumnos adolescentes estudiantes de FPB que han mostrado una menor interferencia, mayor capacidad de planificación y mayor flexibilidad, han tenido un rendimiento académico mayor en el ámbito científico-tecnológico. En cambio, no se ha encontrado influencia de la empatía/afectividad sobre el rendimiento académico.

Recientes investigaciones como la de Zorza (2016), han revelado que la empatía y las habilidades sociales juegan un papel mediador importante sobre el rendimiento académico, sin embargo, en su estudio observaron que los procesos inhibidos evaluados con la tarea STROOP no predijeron la empatía o el comportamiento social. Otra línea de investigación reveló que tener empatía no ejercía una influencia significativa en el nivel de las calificaciones escolares, ya que Freitas (2015) no encontró correlaciones significativas en la prueba TECA con el rendimiento del alumnado, verificándose, sin embargo, que cuanto menor era la empatía (TECA total), mayores eran los resultados de los varones en las matemáticas.

No obstante, la dimensión cognitiva y afectiva de la empatía parece estar estrechamente relacionada con la inteligencia emocional (Gorostiaga et al., 2013). En su trabajo, las personas con buena atención a sus emociones extrapolaron la habilidad al campo interpersonal, lo que facilitó y mejoró su capacidad adaptativa y relacional. El alumnado que mostró una actitud más sensible, empática y colaborativa, es decir, aquel que dispuso de mayores habilidades sociales, obtuvo un rendimiento académico superior con respecto al alumnado que mostró actitudes asociales (Gutiérrez y Expósito, 2015). Sin embargo, no se encontró relación directa entre la inteligencia emocional y el rendimiento académico, dado que valoraron imprescindible contemplar el método, los instrumentos y el análisis estadístico realizado, así como otras variables. Estos resultados concuerdan con los hallados en el presente estudio, donde no ha sido posible determinar la relación existente entre la empatía (adopción de perspectivas, comprensión emocional, estrés empático, alegría empática) y el rendimiento académico (ámbito sociolingüístico y científico-tecnológico).

Esta investigación evidencia la relación entre las funciones ejecutivas (interferencia, flexibilidad y planificación) y el rendimiento académico (ámbito científico-tecnológico). La hipótesis de partida planteaba que las funciones ejecutivas y la empatía influían en el rendimiento académico. Atendiendo a los resultados obtenidos, vemos que dicha hipótesis se 
ha cumplido parcialmente, es decir, se ha encontrado influencia de las funciones ejecutivas en el rendimiento académico (científico-tecnológico). Así pues, los resultados obtenidos permiten establecer un perfil del alumnado que ha logrado un mejor rendimiento a partir de las variables cognitivas analizadas, observando una correlación significativa entre las calificaciones en el ámbito científico-tecnológico y las tres variables de las funciones ejecutivas. Los alumnos que han obtenido calificaciones más altas han presentado menos interferencias, han tenido mayor flexibilidad cognitiva y mayor capacidad de planificación. El alumnado que mostró menor capacidad de flexibilidad cognitiva obtuvo calificaciones más bajas.

Nuestros resultados concuerdan con los encontrados en varias investigaciones como la de De los Ángeles (2012), donde hallaron correlaciones significativas y negativas entre la flexibilidad cognitiva y el rendimiento en matemáticas, es decir, el alumnado que alcanzó un rendimiento académico más alto, tuvo una mayor capacidad de inhibición. Fonseca, Rodríguez y Parra (2016) también encontraron relaciones significativas entre las FE y el rendimiento académico, observando un mayor rendimiento a medida que aumentaba la edad del alumnado en la planificación, la flexibilidad cognitiva y el control inhibitorio, poniendo de manifiesto así la importancia del neurodesarrollo y la importancia de los procesos cognitivos superiores en el aprendizaje. Otras líneas de investigación como las de Monette, Bigras y Guay (2011) o Simmons, Willis y Adams (2012) respaldaron a través de sus estudios que el rendimiento académico parecía estar muy relacionado con algunas funciones ejecutivas frías como la memoria de trabajo y la flexibilidad cognitiva.

La literatura sugiere que en la infancia tardía está más desarrollada la memoria de trabajo, la memorización estratégica, y la planificación visoespacial y durante la última etapa de la adolescencia, se termina de desarrollar la capacidad de planificación por secuencias (Flores et al., 2014). A través de su estudio, mostraron que el desempeño máximo en la Torre de Hanoi se alcanzó hacia los 15 años de edad, afirmando que de las FE intermedias, la planificación era la que más tarde alcanzaba su máximo desarrollo. No obstante, aún falta información sobre la forma en cómo se integran entre ellas (sobre todo durante la adolescencia) y cómo se integran con otras capacidades cognitivas a lo largo del desarrollo. Ya que como afirman Canet, Introzzi, Andrés, y Stelzer (2016), en el acceso a la información, entran en juego varios procesos cognitivos: el proceso de planificación, el control cognitivo 
basado en la intencionalidad y la autorregulación, el proceso atencional, el mantenimiento de la actividad cognitiva, así como la selección y mantenimiento de la atención.

Los resultados obtenidos en el presente estudio invitan a considerar la importancia de las funciones ejecutivas en adolescentes y concretamente, en el alumnado de FPB, a fin de mejorar su rendimiento académico y comprender su curso de desarrollo. Es importante estudiar cómo los niños y los adolescentes son capaces de combinar dichas habilidades con el objetivo de predecir comportamientos de participación, un buen rendimiento en la escuela, la planificación de su futuro o el desarrollo de relaciones sociales significativas (Crone y Steinbeis, 2017).

No obstante, los datos obtenidos en el presente estudio hay que interpretarlos con cautela ya que se presentan una serie de limitaciones como, por ejemplo, la representatividad de la muestra a nivel nacional, es decir, la muestra solo ha sido seleccionada de una sola comunidad autónoma (País Vasco) y no es posible extrapolar los resultados a nivel nacional. También es importante tener en cuenta el instrumento aplicado para evaluar la dimensión emocional, ya que la prueba TECA, solo evalúa la empatía emocional y afectiva, no contemplando otros aspectos emocionales que son igualmente importantes. Posiblemente, los resultados obtenidos puedan deberse a ello (no existiendo relación de esta variable ni con el rendimiento académico, ni con las funciones ejecutivas). Sería interesante hacer una comparación de los resultados obtenidos con alumnos de otras etapas educativas como estudiantes de secundaria o bachillerato. No fue posible medir el CI de los alumnos participantes, aunque ninguno de los alumnos incluidos fue diagnosticado con discapacidad intelectual.

Los hallazgos sugieren que los límites entre las funciones ejecutivas frías y cálidas son bastante permeables ya que las funciones ejecutivas en sí regulan los procesos cognitivos y emocionales y es que, según Pfeifer y Allen (2012), aunque se demuestre que el cerebro y el comportamiento siguen trayectorias de desarrollo similares para una función dada, se debería considerar que una trayectoria cause la otra. En cualquier caso, si se tiene en cuenta la dimensión emocional, social y física de la persona, se estará trabajando hacia la excelencia académica desde una perspectiva inclusiva, capaz de contribuir en la motivación hacia el aprendizaje y en el éxito académico del alumnado de FPB (Sarceda, Santos y Sanjuán, 2017). 
En conclusión y según los resultados obtenidos, las funciones ejecutivas y la empatía no parecen estar relacionadas (son independientes) en adolescentes de FPB. Además, tener un alto desarrollo de las funciones ejecutivas, parece asegurar un buen rendimiento académico.

\section{Referencias}

Army Individual Test Battery (1944). Trail Making Test. Manual of directions and scoring. Waschington DC: War Department, Adjuntant General's Office.

Bausela, E. (2014). Funciones ejecutivas: nociones del desarrollo desde una perspectiva neuropsicológica. Acción Psicológica. 11(1), 21-34. doi:10.5944/ap.1.1.13789

Canet, L., Introzzi, I., Andrés., M.L. y Stelzer, F. (2016). La contribución de las Funciones Ejecutivas a la Autorregulación. Cuadernos de Neuropsicología Panamerican Journal of Neuropsychology, 10 (2), 106-128. doi: 10.7714/CNPS/10.2.206

Charles, J. y Golden, P.H. (1994). Stroop. Test de Colores y Palabras. Madrid: Tea.

Crone, E.A. y Dahl, R.E. (2012). Understanding adolescence as a period of social-affective engagement and goal flexibility. Nature Reviews Neuroscience, 13(1), 636-650. doi: $10.1038 / \mathrm{nrn} 3313$

Crone, E. A., y Steinbeis, N. (2017). Neural perspectives on cognitive control development during childhood and adolescence. Trends in Cognitive Sciences, 21(3), 205-215. doi: 10.1016/j.tics.2017.01.003

Cunningham, W. A., y Zelazo, P. D. (2007). Attitudes and evaluations: A social cognitive neuroscience perspective. Trends in Cognitive Sciences, 11(1), 97-104. doi: 10.1016/j.tics.2006.12.005

De los Ángeles, M. (2012). Las funciones ejecutivas cálidas y el rendimiento académico. (Tesis doctoral). Universidad Complutense de Madrid, Madrid.

Diamond, A. (2013). Executive Functions. Annual Review of Psychology, 64 (1), 135-168. doi:10.1146/annurev-psych-113011-143750.

Flores, J.C., Castillo, R.E. y Jiménez, N.A. (2014). Desarrollo de funciones ejecutivas, de la niñez a la juventud. Anales de psicología, 30(2), 463-473. doi: 10.6018/analesps.30.2.155471

Fonseca, G.P., Rodríguez, L.C. y Parra, J.H. (2016). Relación entre funciones ejecutivas y rendimiento académico por asignaturas en escolares de 6 a 12 años. Hacia la promoción de la salud, 21(2), 41-58. doi:10.17151/hpsal.2016.21.2.4 
Freitas, M.I. (2015). Personalidad, inteligencia emocional y diferencias de género en el rendimiento académico de estudiantes de educación secundaria. (Tesis doctoral). Universidad de León, León.

García, A., Tirapu, J., Luna, P., Ibañez, J., y Duque, P. (2010). ¿Son lo mismo inteligencia y funciones ejecutivas? Revista de Neurología, 50(1), 738-746.

Gorostiaga, A., Balluerka, N. y Soroa, G. (2013). Evaluación de la empatía en el ámbito educativo y su relación con la inteligencia emocional. Revista de Educación, 364(1), 12-38. doi: 10.4438/1988-592X-RE-2014-364-253.

Gutiérrez, M. y Expósito, J. (2015). Autoconcepto, dificultades interpersonales, habilidades sociales y conductas asertivas en adolescentes. Revista de orientación y Psicopedagogía, 26 (2), 42-58. doi: 10.5944/reop.vol.26.num.2.2015.15215.

Ibarrola, B. (2013). Aprendizaje emocionante. Barcelona, España: Siegel.

Jiménez, M.I., y López, E. (2009). Impacto de la Inteligencia Emocional Percibida, Actitudes Sociales y Expectativas del Profesor en el Rendimiento Académico. Electronic Journal of Research in Educational Psychology, 11(1), 075-098.

León, J. (1997). Batería Neuropsicológica Sevilla: Torre de Hanoi. Madrid: Tea.

Ley Orgánica 8/2013, de 9 de diciembre, para la mejora de la calidad educativa. Boletín Oficial del Estado, 295, de 10 de diciembre de 2013.

López-Pérez, B., Fernández-Pinto, I. y Abad, F.J., (2008). Test de Empatía Cognitiva y Afectiva (TECA). Madrid: TEA Ediciones.

López, M., Arán, V., y Richaud, M.C. (2014). Empatía: algunos debates en torno al concepto. Avances de Psicología Latinoamericana, 32 (1), 37-51. doi: http://dx.doi.org/10.12804/ap132.1.2014.03

Miyake, A., Friedman, N. P., Emerson, M. J., Witzki, A. H., Howerter, A. y Wager, T. D. (2000). The unity and diversity of executive functions and their contributions to complex frontal lobe' tasks. A latent variable analysis. Cognitive Psychology, 41(1), 49-100. Doi: 10.1006/cogp.1999.0734

Monette, S., Bigras, M. y Guay, M.C. (2011). The role of the executive functions in school achievement at the end of Grade 1. Journal of Experimental Child Psychology, 109(2), 158-73. doi: 10.1016/j.jecp.2011.01.008

Olmos, P. (2014). Competencias básicas y procesos perceptivos: factores claves en la formación y orientación de los jóvenes en riesgo de exclusión educativa y sociolaboral. Revista de Investigación educativa, 32(2), 531-546. doi: 10.6018/rie.32.2.18155 
Portellano, J.A. (2014). Neuropsicología de la atención, las funciones ejecutivas y la memoria. Madrid, España: Síntesis.

Pérez, L. y Beltrán, J. (2014). Estrategias de aprendizaje: función y diagnóstico en el aprendizaje adolescente. Padres y maestros, 358 (1), 34-38. doi: http://dx.doi.org/pym.i358.y201

Pfeifer, J. y Allen, N. (2012). Arrested development? Reconsidering dual-systems models of brain function in adolescence and disorders. Trends in Cognitiva Sciences, 16 (6), 322-329. doi: 10.1016/j.tics.2012.04.011.

Prencipe, A., Kesek, A., Cohen, J., Lamm.C., Lewis, M.D., y Zelazo, P.D. (2011). Development of hoy and cool executive function during the transition to adolescence. Journal of Experimental Child Psychology, 108 (1), 621-637. doi: 10.1016/j.jecp.2010.09.008

Ritacco, M. y Amores, F. J. (2015). De los programas de Cualificación Profesional Inicial (PCPI) como medida de prevención del fracaso escolar a la Formación Profesional Básica. Un estudio sobre el éxito y fracaso de alumnos en riesgo de exclusión educativa en Educación Secundaria. Revista de Investigación en Educación, 13(1), 105-120. doi: http://dx.doi.org/10.14201/et201634113716.

Salguero, J., Fernández, P., Ruíz, D., Castillo, R. y Palomera, R. (2011). Inteligencia Emocional y ajuste psicosocial en la adolescencia: el papel de la percepción emocional. European Journal of Educational Psychology, 4 (2), 143-152.

Sarceda, M.C., Santos, M.C. y Sanjuán, M.M. (2017). La formación Profesional Básica: ¿alternativa al fracaso escolar? Revista de educación, 378 (1), 78-102. doi: 10.4438/1988-592X-RE-2017-378-362.

Sierra, O., Urrego, G., Montenegro, S. y Castillo, C. (2015). Estrés escolar y empatía en estudiantes de bachillerato practicantes de Mindfulness. Cuadernos de Lingüística Hispánica, 26 (1), 175-197.

Simmons, F.R., Willis, C. y Adams, A.M. (2012). Different components of working memory have different relationships with different mathematical skills. Journal of Experimental Child Psychology, 111 (2), 139-155. doi: 10.1016/j.jecp.2011.08.011.

Zorza, J. P. (2016). Relevancia de las funciones ejecutivas, el Effortful Control y la empatía en el desempeño social y académico de adolescentes. (Tesis doctoral). Universidad de Granada, Granada. 